\title{
Détermination du plutonium dans des excretas une méthode adaptable aux incidents de masse
}

\author{
L. FONTENIL, M. LAFON, O. PEYRONNET, C. ROTGERS * \\ (Manuscrit reçu le 29 décembre 1988)
}

\begin{abstract}
RÉSUMÉ
Nous proposons une méthode de mesure du plutonium dans les fèces et les urines adaptable à diverses situations. Après minéralisation et reprise en milieu acide des échantillons, on réalise une extraction par solvant organique des complexes du Pu IV avec l'acide di-2-(éthylhexyl) phosphorique (Di-2-EHPA). Une extraction en retour de $\mathrm{Pu}$ III en phase aqueuse peut être ensuite réalisée en utilisant la tertiobutylhydroquinone (TBHQ) comme agent réducteur. A ce niveau, il est facile d'obtenir des sources suffisamment purifiées pour comptage.

Selon les circonstances (radiocontamination de masse, surveillance périodique de personnels, phase d'excrétion lente ou rapide...) la purification de l'échantillon peut s'arrêter à des étapes plus ou moins précoces, qui détermineront le mode de comptage (scintillation liquide ou compteur proportionnel).
\end{abstract}

Du fait de sa facilité de mise en œuvre, cette technique peut être pratiquée par des techniciens peu entraînés.

\section{SUMMARY}

With respect to different situations, a versatile method of plutonium determination in feces or urines is described. After a previous treatment involving mineralization and subsequent acidic dissolution, organic solvent extraction of Pu IV complexes with (di-2-ethylhexyl) phosphoric acid (Di-2-EHPA) is used. Aqueous stripping of $\mathrm{Pu}$ III from the organic phase can be performed using tertiobutylhydroquinone (TBHQ) as reducing reagent. At this stage, enough purified samples may be easily obtained for counting purposes. Depending on circumstances (radioactive contamination of large groups, periodic personal monitoring, rapid or large excretion rate period...) sample purification can be stopped at various stages that will decide the "ad-hoc" counting mode (liquid scintillation or proportional counting).

Owing to its feasibility, this technique can even be performed by poorly trained technicians.

- Centre de recherches du service de santé des armées : 24, av. des Maquis du Grésivaudan, B.P. 87, 38702 La Tronche Cedex. 


\section{INTRODUCTION}

La technique de dosage des isotopes du plutonium dans les excrétas pratiquée au Centre de recherches du service de santé des armées depuis les années 1970 est la technique de TALVITIE [9]. Les laboratoires d'analyses médicales du Commissariat à l'énergie atomique (CEA) utilisent la méthode de HENRY [3]. Ces deux techniques, utilisant la fixation de complexes anioniques (nitriques ou chlorhydriques) de $\mathrm{Pu}$ IV sur résines échangeuses d'ions, présentent des rendements chimiques voisins de $90 \%$. Les facteurs de décontamination des autres émetteurs alpha et du fer sont remarquablement élévés. Cependant, elles ne peuvent être mises en œuvre que par des techniciens entraînés du fait de traitements méticuleux : préparation des résines, remplissage de colonnes, débits... Ces mêmes raisons font qu'en régime de travail normal on ne peut, sur urines comme sur fèces, espérer plus de 20 examens par technicien et par semaine.

Pour ces mêmes raisons et en vue de faire face à des situations critiques (incidents de masse) nous avons tenté de mettre en œuvre une technique d'extraction liquide-liquide de complexes de $\mathrm{Pu}$ IV avec l'acide di-2(éthylhexyl) phosphorique (Di-2-EHPA). Les propriétés complexantes de ce composé vis à vis des lanthanides et des actinides sont connus depuis les années 60 [8], mais la qualité des produits commerciaux nécessitait, à l'époque, une purification préalable [6].

Dans les années 70, apparurent diverses techniques de dosage de $\mathrm{Pu}$ utilisant la scintillation liquide après extraction en milieu organique des complexes de Pu IV avec Di-2-EHPA et ajout de molécules fluorescentes $[5,2]$. Ces techniques, peu spécifiques (Th, $U, \mathrm{~Np}, \mathrm{Cm}$ sont extraits) et peu sensibles, du fait du bruit de fond lié à ce type de comptage, peuvent cependant être intéressantes en situation critique, par leur facilité de mise en œuvre. Mme GUSMINI [7] a utilisé Di-2-EHPA couplé aux résines échangeuses d'ions pour fractionner les transuraniens dans des matériaux minéraux.

En 1983, R.P. BARNABEE [1] utilise l'effet réducteur de la 2-5 di-tertiobutylhydroquinone, associée à l'hydrazine et l'acide ascorbique, pour réduire Pu IV à la valence III à partir des complexes formés et le transférer de la phase organique en solution nitrique.

\section{GÉNÉRALITÉS SUR LA MÉTHOdE PROPOSÉE}

Dans le but d'éviter la complexité du mélange précédent, nous proposons une technique utilisant la tertiobutylhydroquinone seule pour réaliser le transfert de $\mathrm{Pu}$ III de la phase organique vers un milieu chlorhydrique. Comme nous le verrons plus loin (paragraphe 7), l'extraction en retour de $\mathrm{Pu}$ III permet d'éliminer ${ }^{232} \mathrm{Th}$ et l'uranium présents dans les selles, ce qui réduit de manière significative le bruit de fond (paragraphes 8.1 et 8.2 ). 
Elle peut être modulée en vue des objectifs suivants :

- surveillance périodique de personnels,

- examens spéciaux,

- tri en cas d'incidents de masse.

Dans sa version la plus complète la radiochimie est terminée par une électrodéposition: à ce propos une cellule jetable, réalisée à partir de matériel de plomberie et de flacons à scintillation liquide en polypropylène est proposée (fig. 1).

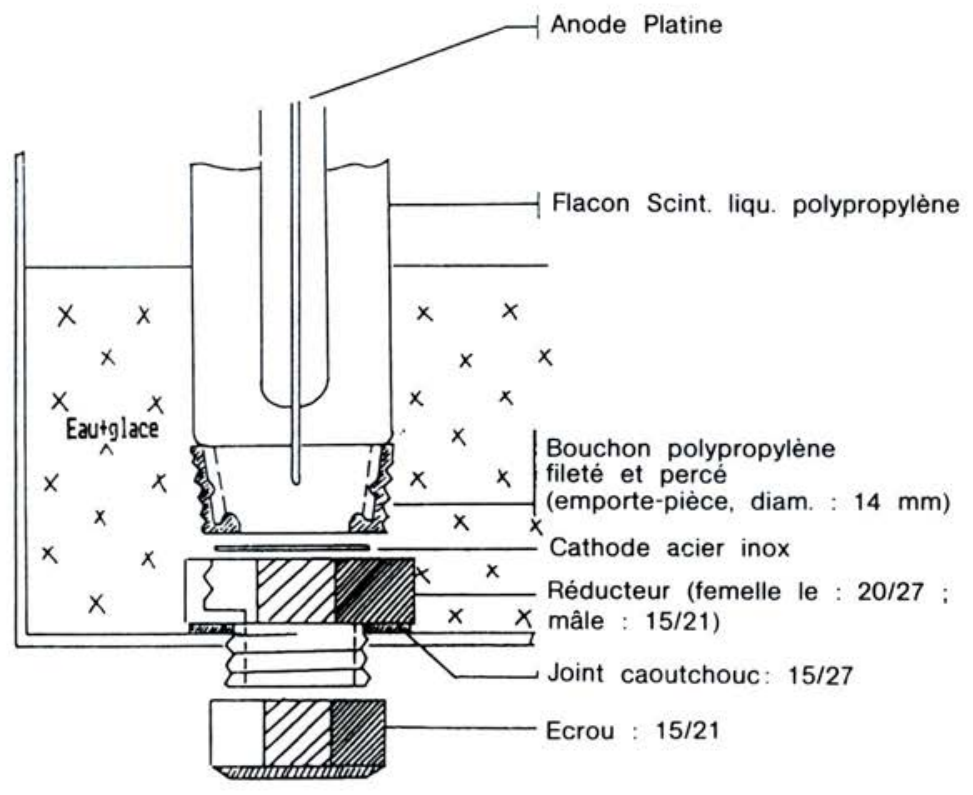

Fig. 1. - Cellule pour électrodéposition (distance anode-cathode: 4 à $5 \mathrm{~mm}$ )

Di-2-EHPA est fourni par BDH (réf. 28135), TBHQ est un réactif FLUKA (réf. 19986, distributeur $\mathrm{OSI}) . \mathrm{HNO}_{3}$ concentré $(\mathrm{d}=1,38), \mathrm{HCl}$ concentré, $\mathrm{NH}_{4} \mathrm{OH}(\mathrm{d}=0,92)$, toluène sont des réactifs purs pour analyse de LABOSI. Le chlorhydrate d'hydroxylamine et le nitrite de sodium sont des réactifs purs PROLABO et le chlorure d'ammonium est un réactif NORMAPUR de - PROLABO. Le mélange scintillant est le PCS d'AMERSHAM. Les cathodes utilisées lors des électrodépositions sont des disques d'acier inox polis sur 1 face de $25 \mathrm{~mm}$ de diamètre, fournis par les établissements Dubois (11, rue de l'Espérance à Rosny-sous-Bois). L'anode est en platine (TACUSSEL XM100). 


\section{3. ÉTUDES DES COEFFICIENTS DE DISTRIBUTION DES COMPLEXES DE PU IV ENTRE PHASE CHLORHYDRIQUE ET PHASE ORGANIQUE}

Les concentrations chlorhydriques varient de 1 à $6 \mathrm{~N}$. Les concentrations de Di-2-EHPA en phase organique (toluène) varient de 0.015 à $0.075 \mathrm{M}$.

On constate (fig. 2 et 3 ):

a/ que le coefficient de distribution $\mathrm{D}=[\mathrm{Pu}$ IV] org/ [Pu IV] aq. décroît lorsque l'acidité croît, atteint un minimum pour $\mathrm{HCl} 4 \mathrm{~N}$ et croit à nouveau (au moins jusqu'à $\mathrm{HCl} 6 \mathrm{~N}$ );

b/ qu'à faible acidité $(1 \mathrm{~N})$ ce coefficient augmente plus rapidement avec la concentration en Di-2-EHPA, comparativement aux autres acidités.

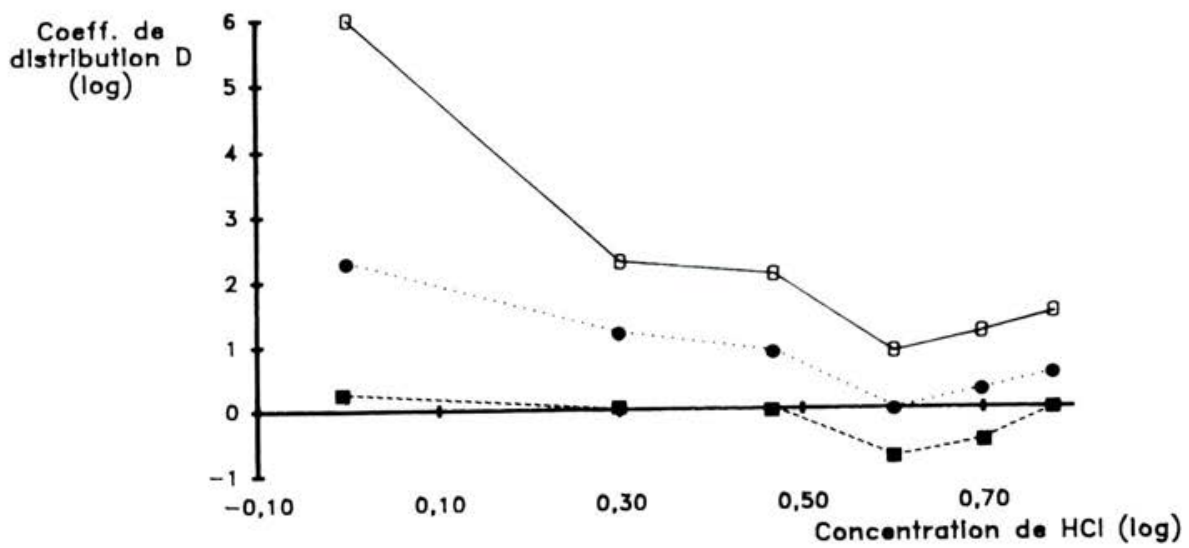

Fig. 2. - Coefficient de distribution en fonction de l'acidité pour trois concentrations en di-2-EHPA (échelle logarithmique):

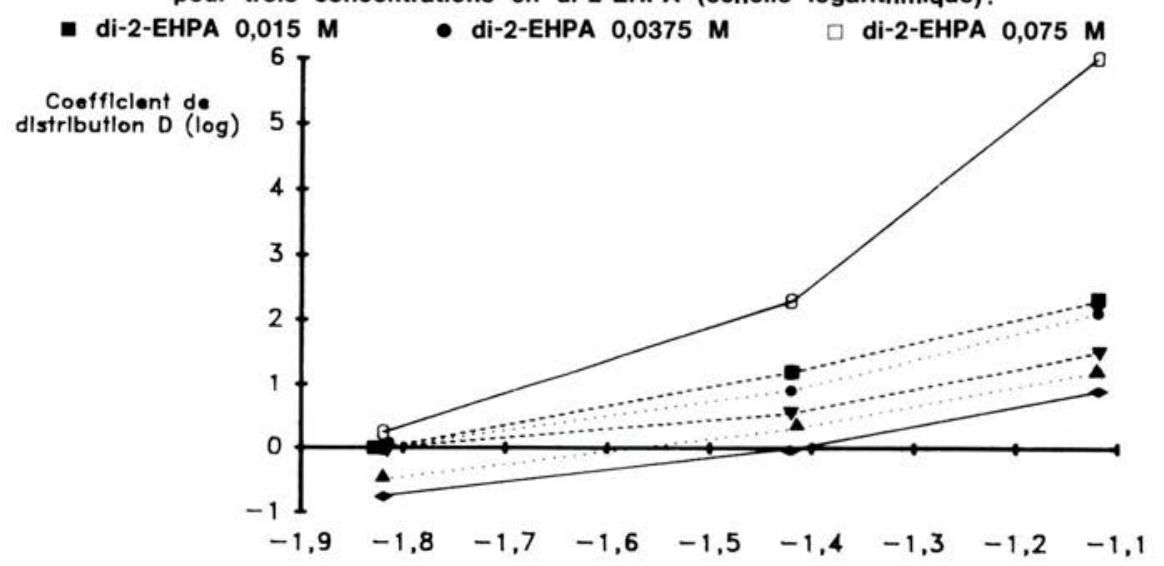

Fig. 3. Concentration en dI-2-EHPA (log)

Fig. 3. - Coefficient de distribution $D$ en fonction de la concentration en di-2-EHPA pour 6 acidités (échelle logarithmique):
$\square \quad 1 \mathrm{~N}$
- $2 \mathrm{~N}$
- $3 \mathbf{N}$
$-4 \mathrm{~N}$
^ $5 \mathrm{~N}$
- $6 \mathrm{~N}$ 


\section{EXTRACTION DE Fe III}

Dans les mêmes conditions, Fe III est toujours extrait, en totalité ou en partie, mais c'est en milieu chlorhydrique $5 \mathrm{~N}$ qu'il est facilement extrait en phase aqueuse, ainsi qu'en témoigne la réaction au thiocyanate d'ammonium.

\section{CONDITIONS D'EXTRACTION DE Pu IV}

Dans l'optique du traitement des minéralisats de selles, le milieu choisi, compromis entre une extraction efficace de Pu IV et la plus faible extraction de Fe III, est $\mathrm{HCl} 6 \mathrm{~N}$ (mélange azéotrope). Le minéralisat de selles est repris par $100 \mathrm{ml}$ de $\mathrm{HCl} 6 \mathrm{~N}$ et soumis à une digestion de $1 / 2 \mathrm{~h}$ dans un bécher couvert. Après refroidissement l'insoluble est décanté, centrifugé dans un tube en polypropylène transféré dans une capsule en platine et soumis à plusieurs attaques fluorhydriques $(10 \mathrm{ml})$ suivies d'évaporation jusqu'à solubilisation totale. Après évaporation, le résidu, dissout dans $\mathrm{HCl}$ à chaud $(10$ à $20 \mathrm{ml})$, est joint à la fraction soluble initiale. Le plutonium est fixé à la valence IV par addition de $\mathrm{NaNO}_{2}$ à $10 \%(1 \mathrm{ml}$, contact $1 / 2 \mathrm{~h})$. II est ensuite extrait dans $10 \mathrm{ml}$ d'une solution au demi de di-2-EHPA dans le toluène (agitation $1 / 2 \mathrm{~h}$ ).

Deux lavages par $100 \mathrm{ml}$ d'une solution chlorhydrique $5 \mathrm{~N}$ de chlorhydrate d'hydroxylamine $0,2 \mathrm{~N}$ sont ensuite réalisés (destruction des composés nitrosés en phase organique, élimination de Fe III).

Un comptage de la phase organique est effectué par scintillation liquide après ajout de $10 \mathrm{ml}$ d'un mélange scintillant. Dans ces conditions, pour 18,5 $\mathrm{Bq}$ de ${ }^{239} \mathrm{Pu}$ ajoutés à la phase initiale, le rendement observé est quasistoechiométrique) non statistiquement différent de $100 \%$ ). Et cela avec des solutions simples de ${ }^{239} \mathrm{Pu}$ dans $\mathrm{HCl} 6 \mathrm{~N}$ ou des minéralisats de selles dissouts dans le même milieu $(100 \mathrm{ml})$.

\section{EXTRACTION EN RETOUR DE Pu III}

Elle a èté réalisée, sur solutions simples ou minéralisats de selles dissouts dans $\mathrm{HCl} 6 \mathrm{~N}$, de la façon suivante:

6.1 Ajout de $10 \mathrm{ml}$ d'une solution saturée de TBHQ dans le toluène à la phase organique. Extraction en retour de Pu III par des solutions chlorhydriques de concentrations variables (1 à $6 \mathrm{~N}$; agitation $1 \mathrm{~h}$ ).

6.2 Évaporation de la phase aqueuse dans un flacon à scintillation liquide en verre. Reprise par $10 \mathrm{ml}$ de $\mathrm{HNO}_{3}$ concentré $(\mathrm{d}=1,38)$ puis évaporation à sec (destruction des traces de matières organiques entraînées).

6.3 Reprise par $5 \mathrm{ml}$ de solution d'HCl $1 \mathrm{~N}$, comptage par scintillation liquide après ajout de $5 \mathrm{ml}$ de mélange scintillant. 
Quels que soient le substrat initial et la concentration de la solution chlorhydrique d'extraction en retour, le rendement chimique moyen est de $95 \%$ avec un écart type de $2,5 \%$.

\section{DÉCONTAMINATION DE THORIUM ET URANIUM}

Ces deux radioéléments naturels, fréquemment présents dans les selles, en particulier le premier (habituellement 40 à $60 \mathrm{mBq}$ ) doivent être aussi complètement éliminés que possible. Divers essais ont été faits avec ${ }^{232} \mathrm{Th}$ (environ $1000 \mathrm{dpm}$ ) et ${ }^{233} \mathrm{U}$ (environ $1200 \mathrm{dpm}$ ).

Les phases d'extraction en retour étaient évaporées, minéralisées par $\mathrm{HNO}_{3}$, puis transférées en quatre fractions de $1 \mathrm{ml}$ de $\mathrm{HNO}_{3}$ concentré, successivement évaporées sur verre de montre pour comptage (compteur proportionnel ENU 15B).

Le coefficient de décontamination de ${ }^{233} \mathrm{U}$ est quasi total puisque, dans les conditions de comptage (36000 s, R $=0,31, \mathrm{BF}: 0,08 \mathrm{cpm}$ ) on n'a pu observer de différence significative avec le bruit de fond.

Pour ce qui concerne ${ }^{232} \mathrm{Th}$ on constate une activité résiduelle variant de moins de $1 \%(\mathrm{HCl} 1 \mathrm{~N})$ à $20 \%(\mathrm{HCl} 6 \mathrm{~N})$. Nous avons donc considéré que le milieu le plus adapté à l'extraction en retour est $\mathrm{HCl} 1 \mathrm{~N}$ pour lequel, dans les conditions habituelles d'élimination, on pourrait s'attendre à récupérer une activité de $0,74 \mathrm{mBq}$ correspondant à 7 coups nets contre 48 de bruit de fond, c'est-à-dire non statistiquement significative.

En utilisant $\mathrm{HCl} 1 \mathrm{~N}$ pour l'extraction en retour, quel que soit le substrat initial (solutions simples ou minéralisats marqués par ${ }^{239} \mathrm{Pu}$ ) le rendement chimique moyen de récupération est de $88 \%$ avec un écart-type de $4 \%$.

\section{PRÉPARATION DE SOURCES POUR COMPTAGE}

On peut envisager, en fonction de la situation, d'arrêter le comptage à quatre stades différents: la figure 4 décrit synthétiquement ces quatre procédures.

\subsection{Scintillation liquide sur phase d'extraction}

Extraction de $\mathrm{Pu}$ IV en phase organique, deux lavages par du chlorhydrate d'hydroxylamine $0,2 \mathrm{~N}$ en solution dans $\mathrm{HCl} 5 \mathrm{~N}$, puis comptage par scintillation liquide après ajout de $10 \mathrm{ml}$ de mélange scintillant. Outre le bruit de fond, relativement élevé, l'élimination de radioéléments naturels fait que cette procédure ne peut se concevoir que lors de la phase d'élimination biologique précoce de ${ }^{239} \mathrm{Pu}$, où l'on peut s'attendre à l'élimination journalière par voie fécale de plus de $4,15 \mathrm{~Bq}(112 \mathrm{pCi})$ à $0,95 \mathrm{~Bq}(26 \mathrm{pCi})$, pendant les cinq premiers jours, pour l'inhalation de $1 / 20^{\mathbf{e}}$ de LAl en classe $Y$ [7]. 


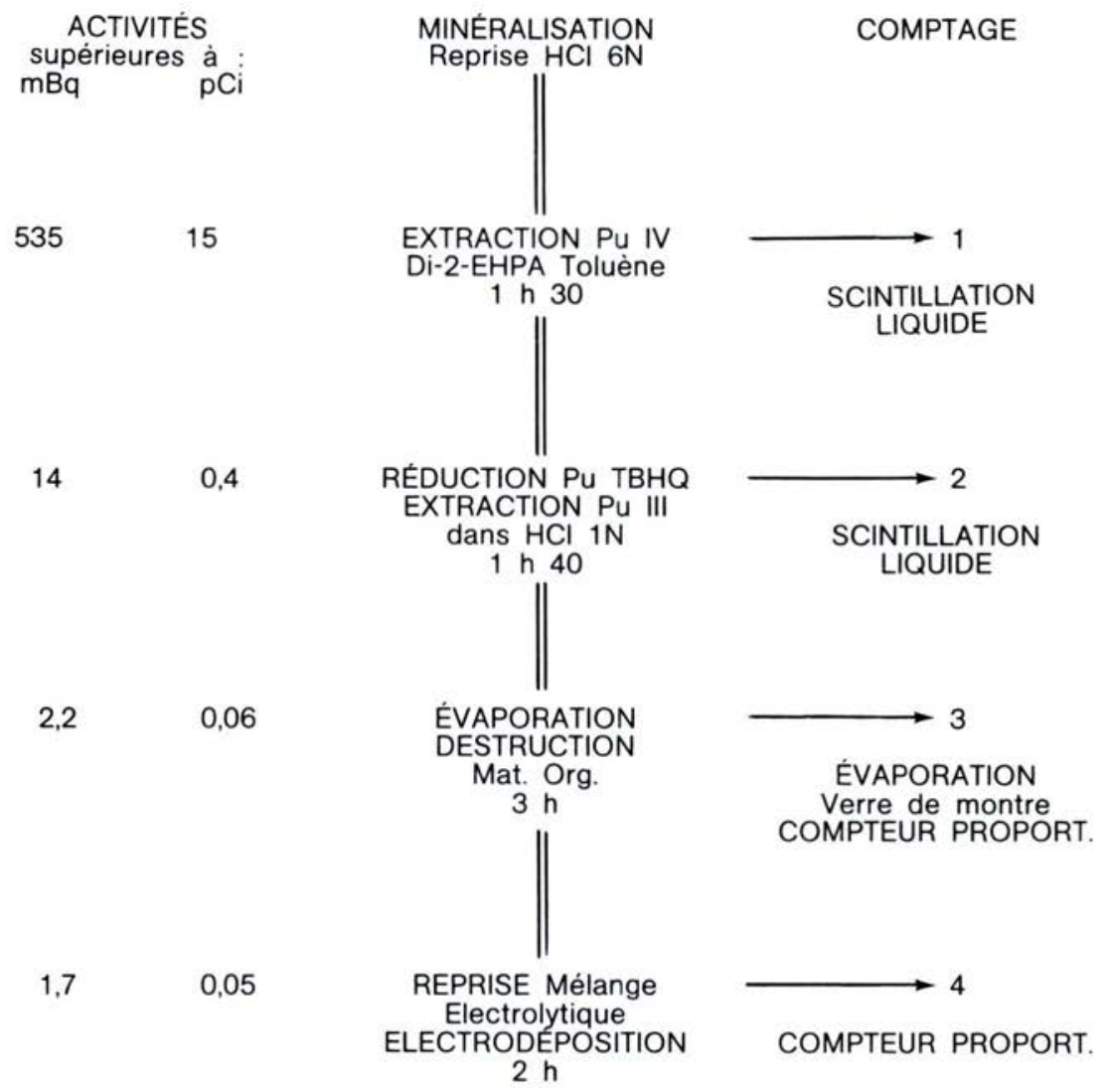

Fig. 4. - Diagramme sommaire des diverses procédures.

Quel que soit le substrat initial (voir ci-après), les mesures de scintillation liquide ont été faites avec une fenêtre conduisant à un rendement de comptage voisin de 0,8 (optimisation du rapport signal/bruit).

La limite de détection dans les conditions habituelles de comptage est de $58 \mathrm{mBq}$ (Packard Biobeta 2002, $6000 \mathrm{~s}, \mathrm{BF}=7 \mathrm{cpm}$ dont $3 \mathrm{cpm}$ de radioactivité naturelle estimée).

\subsection{Scintillation liquide après extraction en retour}

Extraction de $\mathrm{Pu}$ IV, extraction en retour après réduction à la valence III par $5 \mathrm{ml}$ de $\mathrm{HCl} 1 \mathrm{~N}$. Incorporation de $5 \mathrm{ml}$ de mélange scintillant, comptage par scintillation liquide. Dans ces conditions, avec le compteur utilisé (PACKARD BIOBETA 2002), le bruit de fond est d'environ $2 \mathrm{cpm}$. 
Six essais réalisés sur des minéralisats de selles contaminés par $18,5 \mathrm{mBq}$ de ${ }^{239} \mathrm{Pu}$ ont été comparés à six témoins de réactifs et six minéralisats de selles non contaminés: ils se sont révélés significativement supérieurs aux deux groupes témoins (tests de MANN et WHITHNEY ou FISHER: risque $1 \%$ ) alors que les deux lots témoins ne différaient pas significativement.

Le seuil de détection est de $14 \mathrm{mBq}(0,4 \mathrm{pCi})$ dans les conditions habituelles de comptage $(6000 \mathrm{~s}, \mathrm{BF}=2 \mathrm{cpm}, \mathrm{R}=0,80$, rendement chimique: 0,95 ).

Dans ces conditions de traitement chimique, $U$ naturel et Th ne pourraient fausser les résultats que s'ils étaient présents à des quantités anormalement élevées. Cette technique ne peut être envisagée qu'en vue d'un tri dans le cas d'incidents de masse après élimination de ${ }^{239} \mathrm{Pu}$ en classe $\mathrm{Y}$, à partir de $\mathrm{J}+2$ jusqu'au delà de $\mathrm{J}+10$ (même dans la phase d'excrétion lente). On devrait, bien entendu renouveler systématiquement les examens positifs par l'une quelconque des deux méthodes qui suivent.

\subsection{Comptage à bas bruit de fond (verre de montre)}

Extraction de $\mathrm{Pu}$ IV en phase organique, extraction en retour par $10 \mathrm{ml}$ $\mathrm{HCl} 1 \mathrm{~N}(10 \mathrm{ml})$ après réduction à la valence III. Lors de la décantation de la phase aqueuse, il est important d'éviter d'entraîner de la phase organique. A cette fin, on laisse décanter au moins $1 / 2 \mathrm{~h}$ avant de séparer la phase aqueuse. Un lavage par $5 \mathrm{ml}$ de $\mathrm{HCl} 1 \mathrm{~N}$ est réalisé, suivi d'une décantation de même durée, et joint à la fraction précédente. Après évaporation une reprise par $10 \mathrm{ml}$ de $\mathrm{HNO}_{3}$ concentré $(\mathrm{d}=1,38)$ est réalisée suivie d'une nouvelle évaporation avant transfert sur verre de montre par quatre fractions successivement évaporées. Le comptage est réalisé au compteur proportionnel: dans ces conditions on atteint sans problème un seuil de détection de $2,2 \mathrm{mBq}(0,06 \mathrm{pCi})$ dans les conditions habituelles de comptage $(E N U 15 B, t=60000 \mathrm{~s}, B F=0,08 \mathrm{cpm}, \mathrm{R}=0,31$, rendement chimique: 0,88$)$.

Cependant, à ces niveaux d'activités, il sera difficile de différencier par spectrométrie ${ }^{239-238} \mathrm{Pu}$ d'autres émetteurs alpha.

Cette technique peut, cependant, être envisagée pour des examens systématiques chez des sujets exposés à une contamination pulmonaire de $\mathrm{Pu}$ en classe $\mathrm{Y}$.

\subsection{Préparation de sources électrodéposées}

8.4.1. Après extraction en retour de Pu III en phase aqueuse, cette dernière est réduite à environ $1 \mathrm{ml}$ sous épiradiateur (éviter l'évaporation totale: formation de produits charbonneux difficiles à minéraliser en phase liquide).

8.4.2. Après ajout de $10 \mathrm{ml}$ de $\mathrm{HNO}_{3}$ concentré $(\mathrm{d}=1,38)$ on évapore sous épiradiateur à environ $1 \mathrm{ml}$. 
8.4.3. On ajoute $0,5 \mathrm{ml}$ de $\mathrm{HClO}_{4}$, on évapore à sec: la matière organique est alors totalement détruite.

8.4.4. Le résidu est repris par $5 \mathrm{ml}$ d' $\mathrm{HCl} 6 \mathrm{~N}$ puis porté à ébullition dans le même flacon couvert d'un verre de montre pendant environ $1 / 2 \mathrm{~h}$. On évapore ensuite sous épiradiateur.

8.4.5. On met en contact $1 / 2 \mathrm{~h}$ dans $0,5 \mathrm{ml}$ d'HCl $6 \mathrm{~N}$ avec de fréquentes agitations.

8.4.6. La solution chlorhydrique est ensuite partiellement neutralisée par ajout de $0,25 \mathrm{ml}$ d'ammoniaque concentrée puis transférée dans la cellule à électrodéposer. Le flacon est ensuite rinçé par deux fois $3 \mathrm{ml}$ d'une solution de $\mathrm{NH}_{4} \mathrm{Cl} 5,5 \mathrm{M}$ dans $\mathrm{HCl} 0,1 \mathrm{~N}$ fraîchement préparée.

8.4.7. L'électrodéposition est réalisée pendant 50 min sous une intensité de 1,2 A. Après ajout de $1 \mathrm{ml}$ d'ammoniaque concentrée (contact 1 min), le courant est coupé en retirant l'anode. La cuve est rapidement vidée puis la cathode rincée par de l'eau distillée et de l'alcool absolu. Elle est ensuite séchée sur plaque chauffante et refroidie avant comptage et spectrométrie éventuelle.

8.4.8. Le rendement chimique observé est $83,3 \%$ (écart type $8,5 \%$ ). Le seuil de détection ( $E N U 15 B, t=60000 \mathrm{~s}, B F=0,05 \mathrm{cpm}, \mathrm{R}=0,33$ ) est de $1,7 \mathrm{mBq}(0,045 \mathrm{pCi})$.

Des mesures réalisées sur vingt échantillons de selles de $24 \mathrm{~h}$ prélevés chez des personnels non exposés ont donné les résultats suivants:

- bruit de fond moyen (36000 s): 30 coups,

-comptage brut moyen: 36 coups (écart-type: 13 coups).

Cependant, lors d'un premier comptage, certains échantillons ont présenté des activités significativement plus élevées $(55,44,64,53$ coups). Une spectrométrie ne montrant pas d'activité significative, ni bien sur de pics identifiés, un deuxième comptage fut réalisé et les résultats se trouvèrent tous réduits $(36,33,39$ et 36 coups respectivement) et, individuellement, compris dans les limites de fluctuation du bruit de fond (loi de POISSON: espérance mathématique: 30 coups, limites de confiance $95 \%$ : 20,25 à 42,8 coups).

Cependant, ces résultats laissent supposer que des descendants de ${ }^{232} \mathrm{Th}$ ou d'uranium naturel à vie relativement courte expliquent la différence entre l'activité moyennne observée et le bruit de fond. Cette différence correspond en équivalent $\mathrm{Pu}$ à $0,7 \mathrm{mBq}(0,02 \mathrm{pCi})$.

Six électrodépositions peuvent être conduites en parallèle avec le même générateur. 


\section{CAS DES URINES}

Le précipité de phosphates urinaires minéralisé est redissout dans $\mathrm{HCl} 6 \mathrm{~N}(100 \mathrm{ml})$, porté à ébullition légère dans un flacon couvert pendant une $1 / 2 \mathrm{~h}$ (hydrolyse d'éventuels pyrophosphates). Après refroidissement, la solution est traitée comme précédemment. Compte tenu des activités auxquelles on peut s'attendre la technique a été conduite jusqu'à électrodéposition. Le rendement chimique moyen est de $83,4 \%$ (écart type $=6,2 \%)$.

Sur urines $(1250 \mathrm{ml})$, prélevées chez des personnels non exposés ("blancs"), les valeurs observées ne sont pas significativement différentes du bruit de fond:

-BF moyen: 30 coups/36000 s,

- comptage brut moyen: 30,5 coups (écart-type $=5,7$ coups).

\section{CONCLUSION}

L'utilisation de di-2-EHPA comme agent chélatant couplé à TBHQ utilisé comme agent réducteur dans le dosage du plutonium permet d'envisager sur les fèces quatre possibilités de fractionnement en fonction de la situation à laquelle on est confronté.

En cas d'incident de masse on utiliserait la première méthode au cours de la phase d'excrétion rapide. La deuxième méthode peut être utilisée dans la phase d'excrétion lente en vue d'un tri, complété chez des sujets dépassant un seuil à déterminer, par des examens spéciaux réalisés selon la technique 3 ou 4. Dans des circonstances exceptionnelles, vue sa facilité de mise en œuvre, cette technique pourrait être pratiquée par des techniciens non entraînés provenant d'autres laboratoires. En situation "normale", les techniques 3 et 4 seront utilisées en particulier pour la surveillance de personnels exposés (examens périodiques ou spéciaux).

Pour ce qui concerne les urines, dans l'ètat actuel de nos travaux, seules les techniques 3 et 4 peuvent être envisagées. La technique 4 est utilisée en routine au laboratoire de radiotoxicologie du CRSSA depuis plus d'un an. Les résultats obtenus lors d'intercomparaisons dans un groupe de travail du CEA (GT1 technique) réunissant les laboratoires d'analyses médicales du CEA, de la COGEMA, d'EDF, le laboratoire de radiobiologie du Centre d'étude nucléaire de $\mathrm{MOL}$ et notre laboratoire, sont satisfaisants et comparables à ceux des autres participants utilisant les techniques d'échange d'ions.

\section{BIBLIOGRAPHIE}

[1] BARNABEE R.P. - A rapid method for the determination of americium-curium, plutonium an thorium in biological an environmental samples. Health Phys., 1983, 44 (6) 688-691.

[2] BOKOWSKI D.L. - Liquid scintillation counting for plutonium in environmental samples. Am. Ind Hyg. J., 1974, 35, 333-344. 
[3] HENRY P. - Métabolisme et toxicologie du plutonium 239, évaluation de la contamination interne chez des personnels professionnellement exposés. Rapport CEA-R-2381, 1963.

[4] GUSMINI S. - Séparation des actinides dans une solution contenant des radioéléments naturels et des produits de fission. J. Radianal. Chem., 1980, 55 (2) 353260.

[5] KEOUGH R.F. et POWERS G.J. - Determination of plutonium in biological material by extraction and liquid scintillation counting. Anal. Chem., 1970, 42 (3) 42094214.

[6] PARTRIDGE J.A et JENSEN R.C. - Purification of bis-(2-ethylhexyl) hydrogen phosphate by precipitation of copper (II) bis - (2-ethylhexyl) phosphate. J. Inorg. Nucl. Chem., 1969, 31, 2587-2589.

[7] PIECHOWSKI J., MENOUX B. - Protocole de surveillance individuelle pour les contaminations par inhalation de radionucléides en milieu professionnel. Rapport CEA-R-5305, 1985.

[8] STEVENSON P.C. et NERVIK W.E. - The radiochemistry of rare earths, scandium, yttrium and actinium. Washington : National academy of sciences, 1961 (Nuclear science series, NAS-NS 3020).

[9] TALVITIE N.A. - Radiochemical determination of plutonium in environmental and biological samples by ion exchange. Anal. Chem., 1971, 43, 1827-1830. 